\title{
OpenCare Project Personal Communication Device
}

\author{
Stefan Wagner \\ Engineering College of Aarhus \\ Computer Technology and Embedded Systems \\ Aarhus, Denmark \\ sw@iha.dk
}

\begin{abstract}
Pervasive healthcare technology is one of the methods for meeting the challenges of an aging population in many western world countries, as well as an expected major shortage of healthcare personnel. The OpenCare Project is an open source infrastructure framework, designed to be used by researchers and corporate developers for developing experimental assistive living prototypes. The Personal Communication Device is an extension of this infrastructure allowing the user to leave his home while continuing the surveillance of his vital signs through sensors, access to medication reminders and other messages, as well as allowing for easy communication and emergency location services. This paper reports on the current work in progress.
\end{abstract}

Keywords-component; pervasive healthcare, pervasive computing, ubiquitous computing, home care, assisted living, infrastructure, sensor networks, vital signs, sensor, GPS, communication, accellerometer, cell phone, Windows Mobile

\section{INTRODUCTION}

The populations of the western world are rapidly getting older, resulting in a much higher incidence of chronic disease and an expected significant increase of the gross domestic product per capita used on health expenditures throughout most countries experiencing this change in the demographic composition $[1,2,3,4,8]$.

The percentage of the U.S. population (to mention the most significant western world country) reaching the age of 80 years and beyond, will in the US increase from $3.3 \%$ in 2000 , to $4.5 \%$ in 2025 , to $8 \%$ in 2050 [ $4,5,8]$. This amounts to an estimated increase of almost 2.5 times as many citizens at or above the age of 80 compared with today's figures.

At the same time that the number of elderly citizens requiring a higher degree of caretaking and service is growing rapidly throughout 2000 to 2050 , the number of people traditionally considered within the working-age, is decreasing accordingly. Today there are around 4.7 working men and women for every retired or elderly adult. By 2025 this will have changed to 3 workers for each elderly. And in 2050 we end up with merely 2.6 active workers for each elderly. This is roughly half of the workforce compared with the year 2000.
The OpenCare Project and Infrastructure is an open source infrastructure project and framework for implementing and testing assisted living $[6,7]$ prototypes and systems, connecting various vendor's sensor hardware and software components with end-users, caretakers, administrative staff and relatives. The framework targets research groups and commercial companies and public institutions, wishing to implement their tailor-made assisted living solutions on an open source platform and framework, without getting locked to a specific vendor or platform, and being able to use a range of existing commercial sensors, along with their own custom-made hardware and software components, and running on different hardware and operating system platforms.

The OpenCare Infrastructure [8] is structured into the Home, Central, Public and Mobile-tier (see figure 1). The Home-tier logically belongs at the end-users home, while Central-tier contains the central server and database, as well as various business-logic code and web services. The Publictier constitutes the interfaces to the stakeholders who may have an interest in the patient's well-being, doctors, nurses, caretakers and relatives etc. The Central and Public-tiers will not be covered in details in this paper.

At the Home-tier we find a touch-screen based stationary computer unit, the Basestation (also called BaseTerminal), which samples and distributes data from all sensor types, including for instance ECG, blood-pressure, weight, automatic medicine dispenser units [6] and others. This device is using a plugged power supply configuration, which was deemed necessary, in order to supply the needed amount of power for keeping several different wireless connections active, processing business logic and user interface tasks, as well as maintaining continuous communication with the Central-tier using one or more broadband connections for fail-safe operation.

Other research teams have advocated using a mobile device, e.g. a cell phone, as the primary or even sole communications platform $[9,10,11]$. This approach has various flaws however, including the limited processing power of the device, the limited band-with and number of communication channels (for instance limiting Bluetooth communication to one sensor at a time, not allowing for 
ZigBee and proprietary protocols etc). The most significant shortcoming of the mobile device approach however, is the limited battery resources. Our measurements indicate that it is not possible to maintain even one day of continuous communication with a Bluetooth-based ECG device. This is of course not in any way acceptable for any kind of in-situ deployment, as the battery should be charged or changed at an unacceptable frequency. Of course, some sensor types might only need to be read at a very infrequent interval, which of course might help the expected battery life expectancy to be more reasonable.

However, it seems evident that a mobile platform is not applicable for a generic platform such as the OpenCare Infrastructure, where many different types of sensors might be applied, and where the sensor equipment vendors might have different communication frequency and band-with needs. Here we need to provide a stable and effective platform.

Of course the stationary platform comes at a cost, namely that of less mobility for the user. The user cannot leave his or her home and be under continuous observation with the present solution.

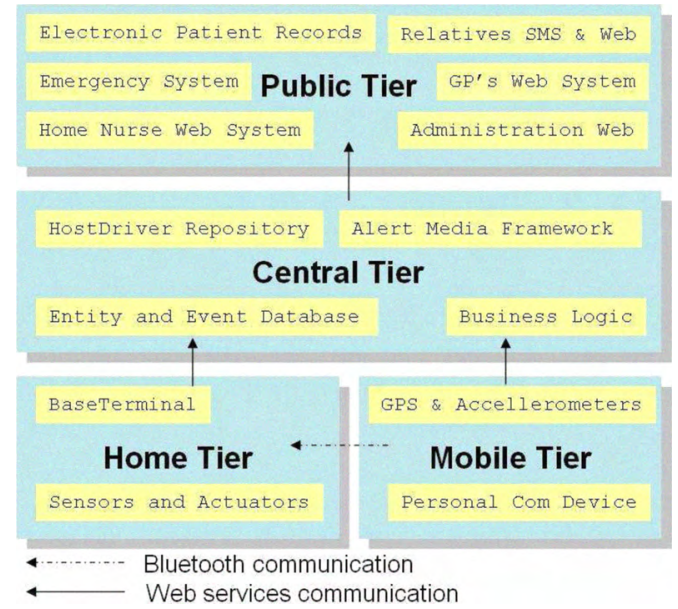

Figure 1. The four tiers of the OpenCare Infrastructure [8], along with the major components contained within them. In the Mobile-tier, we find the Personal Communication Device, including easy cell phone access, Bluetooth sensor communicaiton capabilities, GPS and accellerometer for movement and location tracking.

\section{THE PERSONAL COMMUNICATION DEVICE}

Thus the current problem facing the OpenCare Infrastructure is the lack of mobility and thus flexibility for users leaving their home. The solution to this is to augment the OpenCare Infrastructure with a device with mobile capabilities.

The Personal Communication Device is a mobile extension to the existing infrastructure. It is situated in the Mobile-tier. It is simply a combination of a very noncomplex interfaced cell phone optimized for elderly and cognitive impaired users (see figures 4), combined with a sensor gateway functionality, an accelerometer as well as a GPS service for location tracking. The device utilizes knowledge of whether the device (and thus the user) is currently at home and therefore within range of the stationary Basestation, or not. At a regular interval the device will check for this. In case the device is at the user's home and within range of the Basestation, it will regularly check for new sensor device drivers on the Basestation, and automatically synchronize compatible drivers. A sample scenario might be, that a new Bluetooth based ECG device has been brought to the users home, e.g. to replace a less accurate or outdated device. The Basestation will automatically discover this new device through its zeroconfiguration capabilities [7], download the device drivers for it, and instantiate communications. Within 10 minutes, the Personal Communication device would have checked with the Basestation for new compatible host drivers, it would have found the new ECG device, and downloaded the drivers for it, but it would not yet have started communication (as the Basestation is currently taking care of this). Once the user leaves his home, the Personal Communication Device will simply perform a take-over of communication with all compatible sensors, including the newly discovered ECG device, and thus the user will be monitored by the Personal Communication Device until returning home, where the Basestation again will assume communications, and thus save the battery resources of the Personal Communication Device (see figures 2 and 3 for the different deployment scenarios).

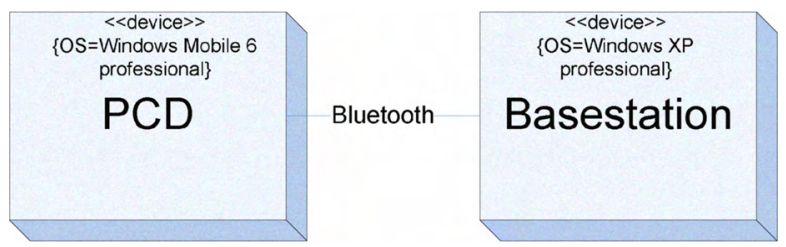

Figure 2. Deployment diagram of the Personal Communication Device (PCD) communicating with the Basestation using Bluetooth. This is both used for synchronizng drivers, checking for location (at home or not), and sending data to the Central-tier.

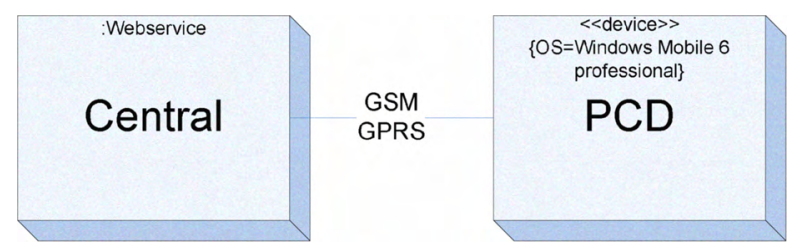

Figure 3. Deployment diagram of PC communications when not in the users home. Here we use the GSM network (or other cell phone networks available) to communicate directly with the Central-tier.

Besides acting as a mobile extension for monitoring and distributing data from the sensors, the device is in itself a sensor, keeping track of the user's movement and activity level using an accelerometer. It also serves as a communications platform, where information, including amongst other reminders and status messages, may be sent to the user wherever he may be located. 


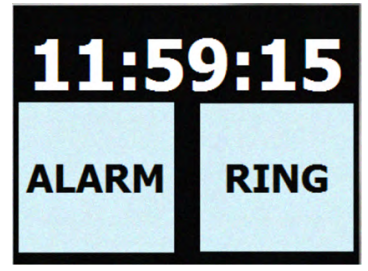

Figure 4. The Personal Screen-shot of the PCD touch-screen, frontpage (default view). Besides showing the time, there are only two menu options for the user. Alert or "Ring"(make a phone call), making the user interface well suited for cognitive impairred users and the elderly.

The device also features voice phone capabilities, with an extremely simple user interface for cognitive impaired users (see figures 4 and 5). Finally, the device features an alert feature, where the user might send an alert in case of getting lost, or having a problem (see figure 4). In case the user is at home (and thus within range of the Basestation) the alert will send the home address (via the Central-tier) to the emergency call-center, and if not, a GPS-reading is attempted, and converted to an address if possible.

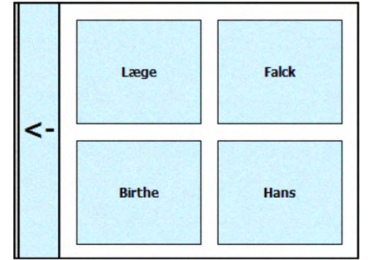

Figure 5. The phone interface (after choosing "Ring"), is extremely simple. The user only has to touch the name that he wants to call, and the call is placed. Instead of using text buttons, images (e.g. with faces) may be used instead.

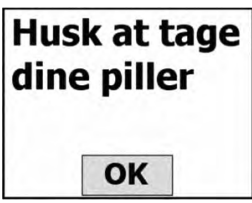

Figure 6. A screen-shot from the PCD. Here a message from the Reminder service, reminding the user to "take his pills".

\section{TECHNOLOGIES}

The PCD is based on the Windows Mobile platform running .NET Compact Framework, using the C\# programming language. The use of SOAP based web services as the primary communication standard ensures a degree of platform independence, including the possibility to use the PCD with other infrastructure systems than the OpenCare Infrastructure, but also allowing for replacing the Windows Mobile platform with another embedded platform.

\section{FUTURE WORK}

Three separate prototypes have been developed by three teams within the OpenCare Project. These have not yet been merged into a single product. The first of these prototypes includes the hand-over sensor communication solution; the second includes support for user friendly phone calls, infrastructure messages (including from the reminder system), and alerts (including GPS support). The third prototype includes the accelerometer and using these data in conjunction with other sensors. These separate products needs to become a unified solution, which is the current focus of the project.

The PCD hardware needs to be further adopted and customized to reduce the size and battery demands. Also, a prototype project trying to equip the PCD with wireless power (for easy and wireless recharging) is currently in progress. A stand-alone working prototype has been constructed.

Finally, it is important to mention that end-user testing has not yet commenced with the system. Usability being a major design criteria for the PCD, this is also of major importance, and is scheduled to commence as soon as a unified prototype is complete.

\section{REFERENCES}

[1] S. Wagner, "Towards an open and easily extendible home care system infrastructure", Proceedings of the 2nd International Conference on Pervasive Computing Technologies for Healthcare, Tampere, Finland, 2008

[2] J.E. Bardram, A. Mihailidis, D. Wan, "Pervasive Computing in Healthcare", CRC Press, 2006.

[3] H. Kautz, L. Arnstein, G. Borriello, O. Etzioni., D. Fox. "An overview of the assisted cognition project.", AAAI-2002 Workshop on Automation as Caregiver: The Role of IntelligentTechnology in Elder Care, Edmonton, Alberta, 2002.

[4] A. Bamis, D. Lymberopoulos, T. Teixeira, A. Savvides, "Towards precision monitoring of elders for providing assistive services". In PETRA 108: Proceedings of the 1st international conference on Pervasive Technologies Related to Assistive Environments, pages 18, New York, NY, USA, 2008. ACM.

[5] US Census Bureau, International Database. Accessed via Internet: http://www.census.gov/ipc/www/idb/, November 2008.

[6] S. Wagner, "Zero-configuration of pervasive healthcare sensor networks" Proceedings of the The Third International Conference on Pervasive Computing and Applications (ICPCA2008), Alexandria, Egypt, 2008

[7] R.A. Soerensen, J.M. Nygaard, "Distributed zero configuration base station", Proceedings of the $2^{\text {nd }}$ International Conference on Pervasive Computing Technologies for Healthcare, Tampere, Finland, 2008

[8] C. Nielsen, S. Wagner, "OpenCare Project: An Open, Flexible and Easily Extendible Infrastructure for Pervasive Healthcare Assisted Living Solutions", Proceedings of the $3^{\text {rd }}$ International Conference on Pervasive Computing Technologies for Healthcare, London, United Kingdom (2009)

[9] H.J. Lee, S.H. Lee, K. Ha, H.C. Jang, W. Chung, J.Y. Kim, et al., "Ubiquitous healthcare service using Zigbee and mobile phone for elderly patients". International Journal of Medical Informatics 2009 3;78(3):193-198

[10] R. Lee, C. Hsiao, C. Chen, M. Liu, "A Mobile-Care System Integrated with Bluetooth Blood Pressure and Pulse Monitor, and Cellular Phone". IEICE Trans.Inf.Syst. 2006 /5/1;E89-D(5):17021711

[11] A. Lorenz, R. Oppermann, "Mobile health monitoring for the elderly: Designing for diversity". Pervasive and Mobile Computing ;In Press, Corrected Proof., 2008 